Article

\title{
Essential Oil Characterization of Thymus vulgaris from Various Geographical Locations
}

\author{
Prabodh Satyal ${ }^{1,2}$, Brittney L. Murray ${ }^{2}$, Robert L. McFeeters ${ }^{2}$ and William N. Setzer ${ }^{2, *}$ \\ 1 Alchemy Aromatic LLC, 621 Park East Blvd., New Albany, IN 47150, USA; prabodhsatyal@gmail.com \\ 2 Department of Chemistry, University of Alabama in Huntsville, Huntsville, AL 35899, USA; \\ blm0029@uah.edu (B.L.M.); robert.mcfeeters@uah.edu (R.L.M.) \\ * Correspondence: wsetzer@chemistry.uah.edu; Tel.: +1-256-824-6519 \\ Academic Editor: Angel A. Carbonell-Barrachina \\ Received: 1 September 2016; Accepted: 24 October 2016; Published: 27 October 2016
}

\begin{abstract}
Thyme (Thymus vulgaris L.) is a commonly used flavoring agent and medicinal herb. Several chemotypes of thyme, based on essential oil compositions, have been established, including (1) linalool; (2) borneol; (3) geraniol; (4) sabinene hydrate; (5) thymol; (6) carvacrol, as well as a number of multiple-component chemotypes. In this work, two different $T$. vulgaris essential oils were obtained from France and two were obtained from Serbia. The chemical compositions were determined using gas chromatography-mass spectrometry. In addition, chiral gas chromatography was used to determine the enantiomeric compositions of several monoterpenoid components. The T. vulgaris oil from Nyons, France was of the linalool chemotype (linalool, 76.2\%; linalyl acetate, $14.3 \%$ ); the oil sample from Jablanicki, Serbia was of the geraniol chemotype (geraniol, 59.8\%; geranyl acetate, $16.7 \%$ ); the sample from Pomoravje District, Serbia was of the sabinene hydrate chemotype (cis-sabinene hydrate, 30.8\%; trans-sabinene hydrate, 5.0\%); and the essential oil from Richerenches, France was of the thymol chemotype (thymol, $47.1 \%$; $p$-cymene, 20.1\%). A cluster analysis based on the compositions of these essential oils as well as 81 additional T. vulgaris essential oils reported in the literature revealed 20 different chemotypes. This work represents the first chiral analysis of $T$. vulgaris monoterpenoids and a comprehensive description of the different chemotypes of T. vulgaris.
\end{abstract}

Keywords: chiral gas chromatography; mass spectrometry; hierarchical cluster analysis; antifungal activity; enantiomeric distribution

\section{Introduction}

Thymus vulgaris L. (Lamiaceae) is an evergreen herb native to the southern Europe and the Mediterranean [1]. The plant has been used since ancient times as a culinary ingredient, to add flavor to cheeses [2,3] and liqueurs [4,5], and to flavor meats such as rabbit, boar, and lamb [6]. Today it is a common component of bouquet garni [7] and of herbes de Provence [8]. In addition to its use in foods, T. vulgaris is a well-known herbal medicine that has been used for thousands of years to treat alopecia, dental plaque, dermatophyte infections, bronchitis, cough, inflammatory skin disorders, and gastrointestinal distress [9]. The major constituents of commercial T. vulgaris essential oil are thymol $(23 \%-60 \%), \gamma$-terpinene $(18 \%-50 \%), p$-cymene $(8 \%-44 \%)$, carvacrol $(2 \%-8 \%)$, and linalool $(3 \%-4 \%)$ [10]. T. vulgaris oil as well as thymol have shown antibacterial, antifungal, and anti-inflammatory effects, accounting for the medicinal uses of T. vulgaris [9]. There are, however, numerous varieties and cultivars of T. vulgaris; Tropicos lists nine subspecies and varieties of T. vulgaris [11]. As many as 13 different chemotypes of T. vulgaris, based on the predominance of particular monoterpenoids in the essential oils, have been identified [12-15]. In this work, we present the essential oil compositions, including monoterpenoid enantiomeric compositions, of four different chemotypes of T. vulgaris essential oils from Europe. In addition, a hierarchical cluster analysis has been carried out to elucidate/delineate the 
various chemotypes of T. vulgaris, and we have examined the antifungal properties of three essential oils from Europe.

\section{Materials and Methods}

\subsection{Plant Material}

Thymus vulgaris \#1, (linalool chemotype), T. vulgaris \#3, (geraniol chemotype), T. vulgaris \#4, (sabinene hydrate chemotype), and T. vulgaris \#5, (thymol chemotype) were collected in May 2015, from Nyons, France $\left(44^{\circ} 21^{\prime} 37^{\prime \prime} \mathrm{N}, 5^{\circ} 8^{\prime} 23^{\prime \prime}\right.$ E), Jablanicki, Serbia $\left(42^{\circ} 59^{\prime} 43^{\prime \prime} \mathrm{N}, 21^{\circ} 55^{\prime} 4^{\prime \prime} \mathrm{E}\right)$, Pomoravje District, Serbia $\left(43^{\circ} 58^{\prime} 0^{\prime \prime} \mathrm{N}, 21^{\circ} 15^{\prime} 0^{\prime \prime} \mathrm{E}\right)$ and Richerenches, France $\left(44^{\circ} 21^{\prime} 37^{\prime \prime} \mathrm{N}, 4^{\circ} 54^{\prime} 47^{\prime \prime} \mathrm{E}\right)$, respectively. The plants were identified by Julien Abisset (Natural Essential SA, France). The air-dried aerial parts of each T. vulgaris sample were subjected to steam distillation for $3 \mathrm{~h}$. After decanting and drying of the oils over anhydrous sodium sulfate, they were stored under refrigeration $\left(-4{ }^{\circ} \mathrm{C}\right)$ until analysis. Aerial parts of T. vulgaris \#1 produced 1.6\% yield of essential oil (EO), T. vulgaris \#3 produced $1.2 \%$ yield, T. vulgaris \#4 produced $1.0 \%$ yield, and T. vulgaris \#5 produced $1.5 \%$ yield.

\subsection{Gas Chromatography—Mass Spectrometry (GC-MS)}

The essential oils of T. vulgaris chemotypes were analyzed by GC-MS using a Shimadzu GCMS-QP2010 Ultra operated in the electron impact $(\mathrm{EI})$ mode (electron energy $=70 \mathrm{eV}$ ), scan range $=40-400 \mathrm{amu}$, scan rate $=3.0 \mathrm{scans} / \mathrm{sec}$, and GC-MS solution software. The GC column was a ZB-5 fused silica capillary column with a ( $5 \%$ phenyl)-polymethylsiloxane stationary phase and a film thickness of $0.25 \mu \mathrm{m}$. The carrier gas was helium with a column head pressure of 80 psi and flow rate of $1.37 \mathrm{~mL} / \mathrm{min}$. Injector temperature was $250{ }^{\circ} \mathrm{C}$ and the ion source temperature was $200{ }^{\circ} \mathrm{C}$. The GC oven temperature program was programmed for $50{ }^{\circ} \mathrm{C}$ initial temperature, temperature increased at a rate of $2{ }^{\circ} \mathrm{C} / \mathrm{min}$ to $260{ }^{\circ} \mathrm{C}$. A $5 \%$ w/v solution of the sample in $\mathrm{CH}_{2} \mathrm{Cl}_{2}$ was prepared and $0.1 \mu \mathrm{L}$ was injected with a splitting mode (30:1). Identification of the oil components was based on their retention indices determined by reference to a homologous series of $n$-alkanes, and by comparison of their mass spectral fragmentation patterns with those reported in the literature [16], and stored in our in-house MS library.

\subsection{Chiral Gas Chromatography-Mass Spectrometry}

Chiral analysis of the essential oils was performed on a Shimadzu GCMS-QP2010S operated in the EI mode (electron energy $=70 \mathrm{eV}$ ), scan range $=40-400 \mathrm{amu}$, scan rate $=3.0 \mathrm{scans} / \mathrm{s}$. GC was equipped with a Restek B-Dex 325 capillary column $(30 \mathrm{~m} \times 0.25 \mathrm{~mm}$ ID $\times 0.25 \mu \mathrm{m}$ film). Oven temperature was started at $50{ }^{\circ} \mathrm{C}$, and then gradually raised to $120^{\circ} \mathrm{C}$ at $1.5{ }^{\circ} \mathrm{C} / \mathrm{min}$. The oven was then raised to $200{ }^{\circ} \mathrm{C}$ at $2{ }^{\circ} \mathrm{C} / \mathrm{min}$ and held for $5 \mathrm{~min}$. Helium was the carrier gas and the flow rate was maintained at $1.8 \mathrm{~mL} / \mathrm{min}$. Samples were diluted $3 \% w / v$ with $\mathrm{CH}_{2} \mathrm{Cl}_{2}$ and then a $0.1 \mu \mathrm{L}$ sample was injected in a split mode with a split ratio of 1:45.

\subsection{Hierarchical Cluster Analysis}

A total of $81 T$. vulgaris essential oil compositions from the published literature, as well as the four compositions from this study were treated as operational taxonomic units (OTUs). The percentage composition of 33 major essential oil components (thymol, $p$-cymene, $\gamma$-terpinene, linalool, carvacrol, geraniol, $\beta$-caryophyllene, cis-sabinene hydrate, borneol, $\alpha$-pinene, terpinene-4-ol, myrcene, 1,8-cineole, $\alpha$-terpineol, camphene, $\alpha$-terpinyl acetate, $\alpha$-terpinene, camphor, limonene, $\beta$-pinene, geranyl acetate, $\alpha$-thujene, geranyl formate, $\beta$-cyclocitral, cis-verbenol, trans-sabinene hydrate, 1-octen-3-ol, thymol methyl ether, caryophyllene oxide, carvacrol methyl ether, bornyl acetate, thymoquinone, and $\alpha$-humulene) was used to determine the chemical relationship between the various T. vulgaris essential oil samples by agglomerative hierarchical cluster (AHC) analysis using the XLSTAT 
software, version 2015.4.01. Pearson correlation was selected as a measure of similarity, and the unweighted pair-group method with arithmetic average (UPGMA) was used for cluster definition.

\subsection{Antifungal Screening}

Antifungal activity was carried out using Candida albicans (ATCC \#18804), Cryptococcus neoformans 24067 (serotype D or var. neoformans), and Aspergillus niger (ATCC \#16888). Qualitative assessment of antifungal activity utilized broth macrodilution (for C. albicans and C. neoformans), whereas minimum inhibitory concentrations (MIC) were determined using microdilution methods. Initially cultures were grown on potato dextrose agar for $48-72 \mathrm{~h}$ before a single colony was isolated and grown in potato dextrose broth for 48-72 hours to create initial liquid cultures. Cells were diluted to a final concentration of $2 \times 10^{3}$ cells $/ \mathrm{mL}$ using MOPS (3-( $N$-morpholino)propanesulfonic acid) buffered RPMI (Roswell Park Memorial Institute) medium and $900 \mu \mathrm{L}$ were aliquoted into sterile $12 \times 75 \mathrm{~mm}$ tubes. Each T. vulgaris essential oil ( $100 \mu \mathrm{L}$ of $1 \%$ DMSO solution) was added to each tube, which were then incubated at $37^{\circ} \mathrm{C}$ for $72 \mathrm{~h}$ in a shaking incubator (175 rpm). For determination of the MIC for C. albicans and C. neoformans, microdilution in 96-well plates was performed in triplicate. Briefly, serial dilution of the T. vulgaris samples was performed by adding $50 \mu \mathrm{L}$ of RPMI to each well then an equal volume of sample to be tested to the first row. After mixing, $50 \mu \mathrm{L}$ was removed and added to the next row. The procedure was repeated for each row. To this mixture, $50 \mu \mathrm{L}$ of cells diluted to $2000 \mathrm{cells} / \mathrm{mL}$ in RPMI were added to each well. The plates were incubated for 48 hours at $37^{\circ} \mathrm{C}$ before growth was quantitated visually based on turbidity.

For the mold-like Ascomycota A. niger, disk diffusion was used to characterize each T. vulgaris essential oil. Initial cultures were grown on malt extract agar for 5-7 days before conidia were collected and suspended in potato dextrose broth. The suspension was then filtered into a sterile test tube using cheesecloth to remove hyphae. Conidia suspension was then diluted until it reached an $\mathrm{OD}_{625}$ of $0.1-0.2$. The suspension $(100 \mu \mathrm{L})$ was plated on malt extract agar before a sterile filter paper disk was placed in the center and $50 \mu \mathrm{L}$ of $T$. vulgaris essential oil was added. The culture was grown for $4-5$ days at $25{ }^{\circ} \mathrm{C}$ before zones of inhibition were determined.

\section{Results and Discussion}

\subsection{Essential Oil Compositions}

The chemical compositions of T. vulgaris essential oils from this study are listed in Table 1. T. vulgaris sample \#1, a linalool chemotype collected from Nyons, France, was dominated by linalool $(76.15 \%)$ and linalyl acetate $(14.26 \%)$. The enantiomeric ratios of these two compounds were 1:99 D/L. Other compounds in the oil were $\beta$-caryophyllene $(2.27 \%)$, camphor $(1.79 \%, 100 \%$ D enantiomer), and camphene $(1.17 \%, \mathrm{D} / \mathrm{L}$ ratio $=99: 1)$. T. vulgaris \#3 was a geraniol chemotype collected from Jablanicki, Serbia, and was rich in geraniol (59.75\%) and esters of geraniol, geranyl acetate $(16.72 \%)$ and geranyl propionate $(1.26 \%)$. Oil \#3 also had sizable concentrations of linalool $(7.15 \%$, D/L ratio $=97: 3$ ) and $\beta$-caryophyllene (3.67\%). T. vulgaris \#4 was collected from Pomoravje District, Serbia, and had sabinene hydrates as defining compounds with cis-sabinene hydrate $(30.77 \%$, D/L ratio $=97: 3)$ and trans-sabinene hydrate $(4.98 \%)$. Other major components in oil \#4 were terpinene-4-ol $(9.50 \%, \mathrm{D} / \mathrm{L}=30: 70)$, linalool $(7.89 \%, \mathrm{D} / \mathrm{L}=3: 97), \gamma$-terpinene $(4.58 \%)$, and myrcene $(4.09 \%)$. T. vulgaris \#5, representing the thymol chemotype, was collected from Richerenches, France. The composition of oil \#5 was rich in thymol (47.06\%) and p-cymene (20.07\%) with lesser quantities of linalool $(5.00 \%$, $\mathrm{D} / \mathrm{L}=1: 99)$ and carvacrol (3.24\%). Interestingly, the L-enantiomer dominated linalool in samples $\# 1, \# 4$, and \#5, but was reversed in sample \#3. Özek and co-workers have found L-linalool to be the predominant enantiomer in several Thymus species, but apparently they did not examine T. vulgaris [17]. Similarly, D-terpinen-4-ol was the major enantiomer in sample \#3, but a minor one in samples \#4 and \#5. An enantiomeric composition analysis of Melaleuca alternifolia revealed this essential oil to be composed of mostly D-cis-sabinene hydrate and D-terpinen-4-ol [18]. D- $\alpha$-Thujene was the dominant enantiomer 
in samples \#3 and \#4, while L- $\alpha$-thujene predominated in sample \#5. The L-enantiomer of $\alpha$-thujene was found to be predominant in various Citrus essential oils [19]. L- $\alpha$-Pinene dominated samples \#1 and \#3, but D- $\alpha$-pinene was the major enantiomer in \#4. Sample \#5 showed a nearly racemic mixture of $\alpha$-pinenes. L-Camphene dominated samples \#1 and \#3, but D-camphene dominated \#4 and \#5. Both $\mathrm{D}-\alpha$-pinene and D-camphene were found to be the predominant enantiomers in Ocimum canum and O. kilimandscharicum essential oils [20]. Remarkably, both D-camphor and D-borneol were the only enantiomers in sample \#1, and D-borneol was also the only enantiomer found in samples \#3 and \#4. However, only L-camphor was found in essential oils \#3 and \#4. This is in contrast to the results of Tabanca and co-workers, who had exclusively observed the L-enantiomers of borneol along with $99 \%$ L-camphor in Micromeria cristata subsp. phrygia [21]. Thus, not only are the chemical profiles different between the different chemotypes, but the enantiomeric distributions are also profoundly different.

Table 1. Chemical compositions and enantiomeric distributions of Thymus vulgaris essential oils.

\begin{tabular}{|c|c|c|c|c|c|c|c|c|c|}
\hline \multirow{2}{*}{ RI } & \multirow{2}{*}{ Compound } & \multicolumn{2}{|c|}{$\# 1$} & \multicolumn{2}{|c|}{$\# 3$} & \multicolumn{2}{|c|}{$\# 4$} & \multicolumn{2}{|c|}{ \#5 } \\
\hline & & $\%$ & $\mathrm{D} / \mathrm{L}$ & $\%$ & $\mathrm{D} / \mathrm{L}$ & $\%$ & $\mathrm{D} / \mathrm{L}$ & $\%$ & $\mathrm{D} / \mathrm{L}$ \\
\hline 752 & 3-Methyl-1-penten-3-ol & $\operatorname{tr}$ & & & & & & & \\
\hline 783 & Methyl $\alpha$-methyl butyrate & 0.10 & & 0.07 & & $\operatorname{tr}$ & & $\operatorname{tr}$ & \\
\hline 850 & (3Z)-Hexenol & & & & & & & 0.05 & \\
\hline 922 & Tricyclene & 0.05 & & $\operatorname{tr}$ & & & & 0.09 & \\
\hline 924 & $\alpha$-Thujene & $\operatorname{tr}$ & & $\operatorname{tr}$ & $91: 9$ & 0.71 & $87: 13$ & 0.43 & $10: 90$ \\
\hline 932 & $\alpha$-Pinene & 0.47 & 1:99 & 0.21 & 1:99 & 1.75 & $85: 15$ & 1.32 & $52: 48$ \\
\hline 947 & $\alpha$-Fenchene & & & & & & & $\operatorname{tr}$ & \\
\hline 948 & Camphene & 1.17 & $99: 1$ & 0.38 & $99: 1$ & 0.25 & $0: 100$ & 1.19 & $10: 90$ \\
\hline 971 & Sabinene & $\operatorname{tr}$ & & 0.05 & $98: 2$ & 2.03 & $78: 22$ & & \\
\hline 977 & 1-Octen-3-ol & 0.43 & & 0.42 & & & & & \\
\hline 977 & 1-Octen-3-ol + $\beta$-Pinene & & & & & 0.97 & $20: 80$ & 0.54 & $20: 80$ \\
\hline 983 & 6-Methylhept-5-en-2-one & & & 0.05 & & & & & \\
\hline 983 & 3-Octanone & & & & & $\operatorname{tr}$ & & $\operatorname{tr}$ & \\
\hline 988 & Myrcene & 0.09 & & 0.44 & & 4.09 & & 1.59 & \\
\hline 996 & 3-Octanol & $\operatorname{tr}$ & & 0.07 & & $\operatorname{tr}$ & & & \\
\hline 1004 & (3Z)-Hexenyl acetate & $\operatorname{tr}$ & & & & & & & \\
\hline 1004 & p-Mentha-1(7),8-diene & & & & & & & $\operatorname{tr}$ & \\
\hline 1006 & $\alpha$-Phellandrene & & & & & $\operatorname{tr}$ & $55: 45$ & 0.11 & \\
\hline 1008 & $\delta$-3-Carene & & & & & & & $\operatorname{tr}$ & \\
\hline 1016 & $\alpha$-Terpinene & & & $\operatorname{tr}$ & & 2.65 & & 1.30 & \\
\hline 1019 & $o$-Cymene & & & & & & & 0.06 & \\
\hline 1024 & p-Cymene & 0.09 & & 0.18 & & 1.09 & & 20.07 & \\
\hline 1028 & Limonene & 0.05 & & 0.39 & $85: 15$ & 2.85 & $86: 14$ & 0.39 & $80: 20$ \\
\hline 1030 & $\beta$-Phellandrene & & & $\operatorname{tr}$ & & 0.37 & & 0.09 & \\
\hline 1031 & 1,8-Cineole & 0.37 & & 0.31 & & 0.30 & & 0.72 & \\
\hline 1033 & m-Cymene & & & & & & & $\operatorname{tr}$ & \\
\hline 1034 & Lavender lactone $+(Z)-\beta$-Ocimene & $\operatorname{tr}$ & & & & & & & \\
\hline 1045 & $(E)-\beta$-Ocimene & $\operatorname{tr}$ & & & & & & 0.05 & \\
\hline 1057 & $\gamma$-Terpinene & & & 0.09 & & 4.58 & & 9.03 & \\
\hline 1063 & 3-Methylbut-2-enyl butanoate & $\operatorname{tr}$ & & & & & & & \\
\hline 1069 & cis-Sabinene hydrate & & & 0.31 & & 30.77 & $97: 3$ & 0.17 & \\
\hline 1070 & cis-Linalool oxide (furanoid) & 0.28 & & & & & & & \\
\hline 1071 & Pinol & & & & & & & $\operatorname{tr}$ & \\
\hline 1084 & Terpinolene & & & & & 0.97 & & 0.07 & \\
\hline 1085 & trans-Linalool oxide (furanoid) & 0.23 & & & & & & $\operatorname{tr}$ & \\
\hline 1089 & p-Cymenene & & & & & & & $\operatorname{tr}$ & \\
\hline 1099 & Linalool & 76.15 & 1:99 & 7.15 & $97: 3$ & 7.89 & 3:97 & 5.00 & 1:99 \\
\hline 1101 & trans-Sabinene hydrate & & & $\operatorname{tr}$ & & 4.98 & & $\operatorname{tr}$ & \\
\hline 1103 & Hotrienol & 0.07 & & & & & & & \\
\hline 1104 & Nonanal & 0.05 & & & & $\operatorname{tr}$ & & & \\
\hline 1106 & $\alpha$-Pinene oxide & & & $\operatorname{tr}$ & & & & & \\
\hline 1107 & 1-Octen-3-yl acetate & $\operatorname{tr}$ & & & & $\operatorname{tr}$ & & & \\
\hline 1124 & cis- $p$-Menth-2-en-1-ol & & & & & 0.65 & & & \\
\hline 1136 & trans-Limonene oxide & & & & & $\operatorname{tr}$ & & & \\
\hline 1142 & trans-p-Menth-2-en-1ol & & & & & 0.25 & & & \\
\hline 1147 & Camphor & 1.79 & 100:0 & 0.11 & $0: 100$ & 0.10 & $0: 100$ & 1.42 & $95: 5$ \\
\hline 1148 & $\alpha$-Cyclogeraniol & & & $\operatorname{tr}$ & & & & & \\
\hline 1154 & $\beta$-Pinene oxide & & & & & 0.05 & & & \\
\hline
\end{tabular}


Table 1. Cont.

\begin{tabular}{|c|c|c|c|c|c|c|c|c|c|}
\hline \multirow{2}{*}{ RI } & \multirow{2}{*}{ Compound } & \multicolumn{2}{|c|}{ \#1 } & \multicolumn{2}{|c|}{ \#3 } & \multicolumn{2}{|c|}{ \#4 } & \multicolumn{2}{|c|}{ \#5 } \\
\hline & & $\%$ & $D / L$ & $\%$ & $D / L$ & $\%$ & $D / L$ & $\%$ & $D / L$ \\
\hline 1162 & Lavandulol & $\operatorname{tr}$ & & $\operatorname{tr}$ & & & & $\operatorname{tr}$ & \\
\hline 1169 & cis-Linalool oxide (pyranoid) & $\operatorname{tr}$ & & & & & & & \\
\hline 1171 & Borneol & 0.40 & 100:0 & 1.00 & 100:0 & 0.28 & 100:0 & 1.50 & $71: 29$ \\
\hline 1174 & trans-Linalool oxide (pyranoid) & $\operatorname{tr}$ & & & & & & & \\
\hline 1178 & p-Mentha-1,8-dien-4-ol & & & & & $\operatorname{tr}$ & & & \\
\hline 1180 & Terpinen-4-ol & 0.06 & & 0.17 & 70:30 & 9.50 & $30: 70$ & 1.25 & $40: 60$ \\
\hline 1184 & (3Z)-Hexenyl butanoate & $\operatorname{tr}$ & & & & & & & \\
\hline 1186 & p-Cymen-8-ol & & & & & & & $\operatorname{tr}$ & \\
\hline 1194 & $\alpha$-Terpineol & 0.11 & $65: 35$ & 0.09 & $65: 35$ & 2.69 & 91:9 & 0.16 & $60: 40$ \\
\hline 1196 & cis-Piperitol & & & & & 0.14 & & & \\
\hline 1197 & cis-Dihydrocarvone & $\operatorname{tr}$ & & $\operatorname{tr}$ & & & & $\operatorname{tr}$ & \\
\hline 1205 & $\beta$-Cyclogeraniol & & & $\operatorname{tr}$ & & & & & \\
\hline 1206 & Decanal & $\operatorname{tr}$ & & & & & & & \\
\hline 1208 & trans-Piperitol & & & & & 0.16 & & & \\
\hline 1217 & 7-Ethylidenebicyclo[3.3.0]octan-3-one & & & & & 0.90 & & & \\
\hline 1220 & 6,7-Epoxyneral & & & $\operatorname{tr}$ & & & & & \\
\hline 1223 & Nerol & $\operatorname{tr}$ & & 0.97 & & & & & \\
\hline 1223 & 7-Methylenebicyclo[3.3.1]nonan-3-ol & & & & & 6.07 & & & \\
\hline 1225 & Citronellol & $\operatorname{tr}$ & & 0.35 & & & & & \\
\hline 1228 & Thymol methyl ether & & & & & & & 0.19 & \\
\hline 1230 & 6,7-Epoxygeranial & & & $\operatorname{tr}$ & & & & & \\
\hline 1234 & 4-t-Amylcyclohexanone & 0.08 & & & & & & & \\
\hline 1237 & Neral & $\operatorname{tr}$ & & 0.61 & & $\operatorname{tr}$ & & & \\
\hline 1237 & Carvacrol methyl ether & & & & & & & 0.32 & \\
\hline 1243 & Carvone & & & $\operatorname{tr}$ & & & & 0.09 & \\
\hline 1249 & Linalyl acetate & 14.26 & 1:99 & & & 3.40 & $0: 100$ & & \\
\hline 1249 & Geraniol & & & 59.75 & & 0.24 & & 0.05 & \\
\hline 1266 & Geranial & $\operatorname{tr}$ & & 1.25 & & $\operatorname{tr}$ & & $\operatorname{tr}$ & \\
\hline 1271 & $(2 E)$-Decenal & $\operatorname{tr}$ & & & & & & & \\
\hline 1279 & Isothymol & & & & & & & 0.07 & \\
\hline 1282 & Lavandulyl acetate & $\operatorname{tr}$ & & & & & & & \\
\hline 1284 & Bornyl acetate & 0.21 & & 0.14 & & 0.07 & & & \\
\hline 1284 & neo-iso-3-Thujanol acetate & & & & & & & $\operatorname{tr}$ & \\
\hline 1288 & Thymol & & & 0.42 & & $\operatorname{tr}$ & & 47.06 & \\
\hline 1296 & Carvacrol & 0.05 & & & & & & 3.24 & \\
\hline 1296 & Geranyl formate & & & 0.09 & & & & & \\
\hline 1331 & 2,3-Epoxygeraniol & & & 0.06 & & & & & \\
\hline 1341 & cis-p-Menthadienyl acetate & & & 0.19 & & 4.75 & & & \\
\hline 1344 & Citronellyl acetate & & & & & 0.28 & & & \\
\hline 1346 & $\alpha$-Terpinyl acetate & & & & & 0.05 & & & \\
\hline 1348 & Citronellyl acetate & & & 0.07 & & & & & \\
\hline 1349 & Eugenol & & & & & & & 0.08 & \\
\hline 1357 & Neryl acetate & $\operatorname{tr}$ & & 0.18 & & $\operatorname{tr}$ & & & \\
\hline 1372 & trans- $p$-Menthadienyl acetate & & & & & 0.05 & & & \\
\hline 1375 & $\alpha$-Copaene & & & & & & & $\operatorname{tr}$ & \\
\hline 1377 & Geranyl acetate & 0.06 & & 16.72 & & 0.48 & & & \\
\hline 1383 & $\alpha$-Bourbonene & 0.20 & & $\operatorname{tr}$ & & 0.09 & & & \\
\hline 1388 & $\beta$-Elemene & $\operatorname{tr}$ & & & & & & & \\
\hline 1403 & Isocaryophyllene & $\operatorname{tr}$ & & $\operatorname{tr}$ & & & & & \\
\hline 1419 & $\beta$-Caryophyllene & 2.27 & & 3.67 & & 2.03 & & 1.79 & \\
\hline 1429 & $\beta$-Copaene & $\operatorname{tr}$ & & & & & & & \\
\hline 1429 & cis-Carvyl propanoate & & & & & 0.30 & & & \\
\hline 1434 & $\begin{array}{l}\text { 7-Methyl-3-methylene-7-octen-1-yl } \\
\text { propanoate }\end{array}$ & & & & & $\operatorname{tr}$ & & & \\
\hline 1437 & Aromadendrene & & & & & & & $\operatorname{tr}$ & \\
\hline 1454 & $\alpha$-Humulene & 0.06 & & 0.12 & & 0.06 & & 0.05 & \\
\hline 1468 & Geranyl propanoate & $\operatorname{tr}$ & & 1.26 & & & & & \\
\hline 1473 & trans-Cadina-1(6),4-diene & & & & & & & 0.06 & \\
\hline 1480 & Germacrene D & 0.26 & & 0.05 & & 0.58 & & & \\
\hline 1489 & Viridiflorene & & & & & & & $\operatorname{tr}$ & \\
\hline 1494 & Bicyclogermacrene & & & $\operatorname{tr}$ & & 0.09 & & & \\
\hline 1497 & $\alpha$-Muurolene & & & & & & & $\operatorname{tr}$ & \\
\hline 1506 & Geranyl isobutyrate & & & 0.15 & & & & & \\
\hline 1506 & $\beta$-Bisabolene & & & & & & & $\operatorname{tr}$ & \\
\hline 1511 & $\delta$-Amorphene & & & & & & & 0.07 & \\
\hline 1516 & $\delta$-Cadinene & $\operatorname{tr}$ & & & & & & 0.16 & \\
\hline
\end{tabular}


Table 1. Cont

\begin{tabular}{|c|c|c|c|c|c|c|c|c|c|}
\hline \multirow{2}{*}{ RI } & \multirow{2}{*}{ Compound } & \multicolumn{2}{|c|}{$\# 1$} & \multicolumn{2}{|c|}{$\# 3$} & \multicolumn{2}{|c|}{ \#4 } & \multicolumn{2}{|c|}{ \#5 } \\
\hline & & $\%$ & $\mathrm{D} / \mathrm{L}$ & $\%$ & $\mathrm{D} / \mathrm{L}$ & $\%$ & $\mathrm{D} / \mathrm{L}$ & $\%$ & $D / L$ \\
\hline 1531 & trans-Cadina-1,4-diene & & & & & & & $\operatorname{tr}$ & \\
\hline 1547 & Elemol & & & 0.96 & & & & & \\
\hline 1554 & Geranyl butanoate & 0.05 & & 0.74 & & & & & \\
\hline 1575 & Germacrene-D-4-ol & 0.10 & & & & $\operatorname{tr}$ & & & \\
\hline 1575 & Spathulenol & & & $\operatorname{tr}$ & & & & & \\
\hline 1581 & Caryophyllene oxide & 0.41 & & 0.50 & & 0.10 & & 0.13 & \\
\hline 1595 & Geranyl isovalerate & & & 0.06 & & & & & \\
\hline 1631 & 10-epi- $\gamma$-Eudesmol & & & $\operatorname{tr}$ & & & & & \\
\hline 1654 & $\alpha$-Eudesmol & & & 0.17 & & & & & \\
\hline
\end{tabular}

\subsection{Chemotypes of Thymus vulgaris}

A total of 85 T. vulgaris essential oil compositions were used to carry out a hierarchical cluster analysis (Figure 1). The cluster analysis revealed as many as 20 different chemotypes. The chemotype with the most samples (39) was the thymol chemotype, which had been recognized previously [12-15]. Other previously recognized chemotypes were the geraniol chemotype, with three representative samples (including sample \#3 from Serbia in this study); the linalool chemotype, with four samples (including sample \#1 from France in this study); the carvacrol chemotype, with two samples; the borneol chemotype, only one sample; the sabinene hydrate/terpinene-4-ol chemotype, with two samples (including sample \#4 from Serbia in this study); and the cyclocitral/verbenol chemotype, with only one sample. The second most populated chemotype in the cluster analysis was a $p$-cymene/thymol type represented by 18 samples. The $\alpha$-terpineol chemotype [12] was not found in the present study; only three T. vulgaris samples (all from Austria) showed $\alpha$-terpineol concentrations around $10 \%$, but all three had higher concentrations of other components [14]. Other chemotypes identified include carvacrol $/ \gamma$-terpinene/thymol, which was previously described as carvacrol and thymol/carvacrol [14]; linalool/carvacrol/ $p$-cymene, previously labeled carvacrol/linalool [14]; sabinene hydrate/geraniol/geranyl acetate/ $\alpha$-terpineol, previously described as geraniol/ $\alpha$-terpineol/sabinene hydrate [14]; sabinene hydrate/ $\alpha$-terpinyl acetate/thymol, previously described as $\alpha$-terpineol/sabinene hydrate/thymol [14]; sabinene hydrate/linalool [14]; $\alpha$-terpinyl acetate/carvacrol, previously described as $\alpha$-terpineol/carvacrol [14]; carvacrol/ $\alpha$-terpineol/borneol; sabinene hydrate/ terpinen-4-ol, which includes one of the samples from France in this study; geranyl formate/geraniol, previously called geraniol [13]; $p$-cymene/ thymol/carvacrol; terpinene-4-ol/ $p$-cymene; camphor/camphene; and 1,8-cineole/ $\alpha$-terpinyl acetate. 
Thymus vulgaris Dendrogram

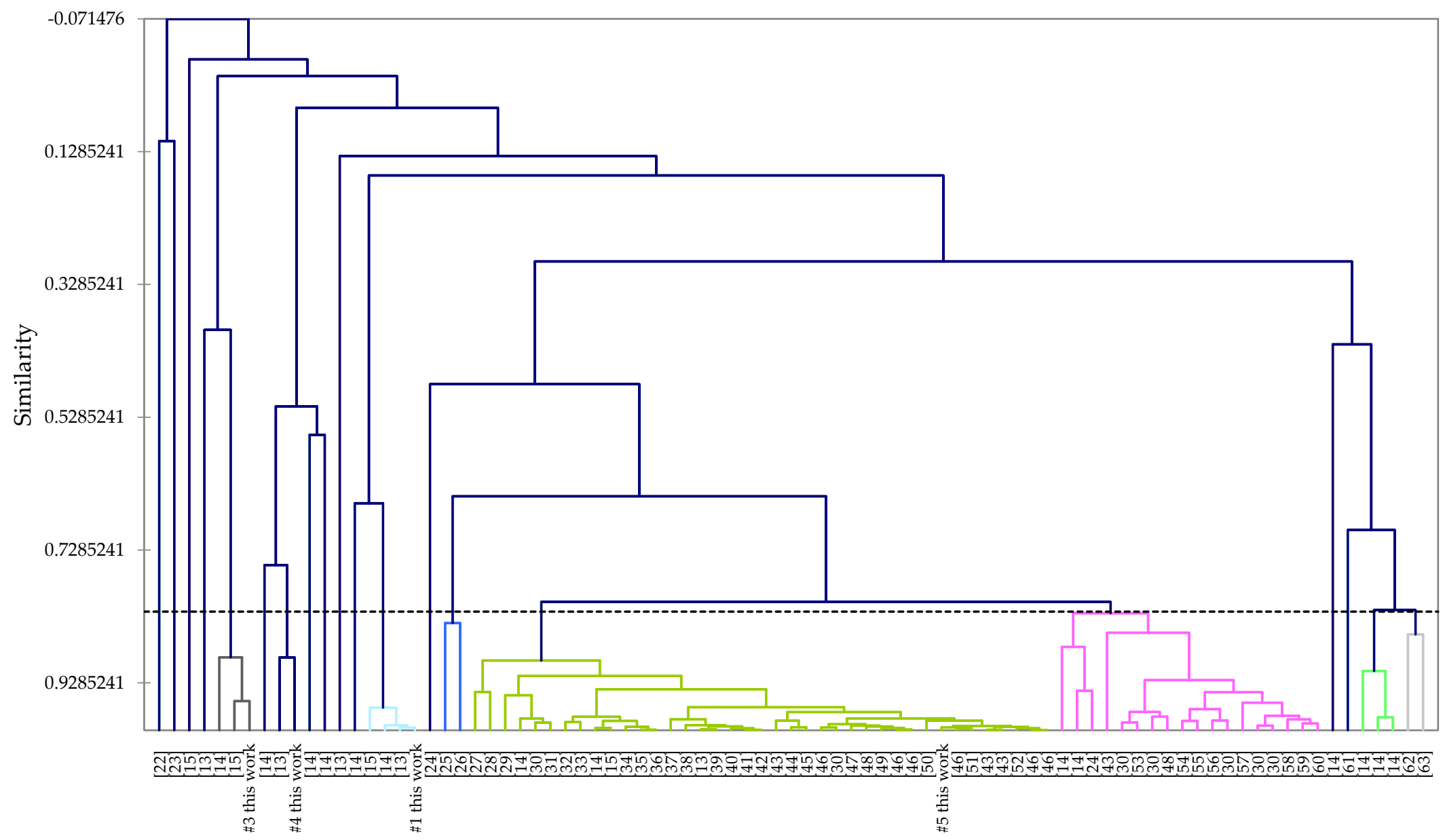

Figure 1. Dendrogram obtained from the agglomerative hierarchical cluster analysis of 85 Thymus vulgaris essential oil compositions. 


\subsection{Antifungal Activity}

Three of the four T. vulgaris chemotypes in this study were tested for inhibition of Aspergillus niger, Cryptococcus neoformans var. neoformans, and Candida albicans. Macrodilution was utilized for the yeast-like Ascomycota, C. albicans, and Basidiomycota C. neoformans, to determine antifungal minimum inhibitory concentrations (MICs, Table 2). The T. vulgaris linalool and geraniol chemotypes both demonstrated some degree of inhibition against these pathogens. For the sporulating mold-like Ascomycota, A. niger, a larger surface area was required for hyphal growth. Thus, it was grown on malt extract agar plates with a filter disk impregnated with the $T$. vulgaris chemotype of interest. Disk diffusion showed only slight inhibition of $A$. niger with the only clear zone of inhibition for T. vulgaris sample \#1. The remaining chemotypes showed growth over the filter disk, indicating no significant antifungal activity against $A$. niger. Because it has been shown that linalool is biotransformed to non-pathogenic compounds and that linalyl acetate increases A. niger hyphal growth [64], it is speculated that camphor in sample \#1 is responsible for $A$. niger inhibition. Significant levels of camphor are not found in the other T. vulgaris samples.

Table 2. Antifungal activities (minimum inhibitory concentrations, MIC) of Thymus vulgaris essential oils.

\begin{tabular}{ccc}
\hline \multirow{2}{*}{ Essential Oil } & \multicolumn{2}{c}{ MIC $(\mu \mathrm{g} / \mathrm{mL})$} \\
\cline { 2 - 3 } & C. albicans & C. neoformans \\
\hline \#1 (linalool chemotype) & 1250 & 313 \\
\#3 (geraniol chemotype) & 625 & 156 \\
\#4 (sabinene hydrate chemotype) & $>2500$ & $>2500$ \\
\hline
\end{tabular}

The differential antifungal activities observed in this study mirror those previously reported by Giordani and co-workers [13]. That is, the sabinene hydrate chemotype showed the lowest antifungal activity, the linalool chemotype was next, then the geraniol chemotype. Giordani and co-workers had found that the thymol chemotype showed much stronger antifungal activity [13]. The T. vulgaris thymol chemotype was also found to be the most larvicidal against Culex quinquefasciatus [15] and exhibited the most antioxidant properties [14].

\section{Conclusions}

This work has presented the most comprehensive analysis of Thymus vulgaris chemotypes, revealing at least 20 different types based on essential oil composition. In addition, this is the first analysis to characterize the enantiomeric distributions of T. vulgaris monoterpenoids. Enantiomers are well known to elicit different odorant responses in insects $[65,66]$ as well as humans [67], and it is reasonable to assume that different enantiomers will have different medicinal biological activities [68]. Thus, for example L-linalool has shown anticonvulsant activity in a mouse model whereas D-linalool was inactive [69]. Similarly, both D- $\alpha$-pinene and D- $\beta$-pinene showed antifungal activity whereas the L-enantiomers were inactive [70], while L- $\alpha$-pinene was more active than the D-enantiomer against Listeria monocytogenes [71]. Therefore, not only is the particular chemotype of a culinary and medicinal herb such as T. vulgaris an important consideration, but the enantiomeric distribution may also have a profound influence on its bioactivity, flavor, and aroma profile.

Acknowledgments: We thank Julien Abisset for plant identification, Joshua Thomerson for assistance with steam distillation of the essential oils, and Erin McClelland (Middle Tennessee State University) for C. neoformans cells. B.L.M. was supported by a GEM University Fellowship.

Author Contributions: P.S. conceived and designed the experiments; P.S. carried out the GC-MS analysis; B.L.M. did the antifungal assays; W.N.S. performed the cluster analysis; R.L.M. and W.N.S. analyzed the data; all authors contributed to writing the manuscript.

Conflicts of Interest: The authors declare no conflict of interest. 


\section{References}

1. Gillett, M. Growing and Using Thyme; Storey Publishing: North Adams, MA, USA, 1998.

2. Aygun, O.; Aslantas, O.; Oner, S. A survey on the microbiological quality of Carra, a traditional Turkish cheese. J. Food Eng. 2005, 66, 401-404. [CrossRef]

3. Akarca, G.; Çağlar, A.; Tomar, O. The effects of spicing on quality of mozzarella cheese. Mljekarstvo 2016, 66, 112-121.

4. Martinez-Francés, V.; Rios, S. Lesser known herbal spirits of the Valencia and Alicante provinces (east-southern Spain). Ind. Crops Rural Dev. 2005, 417-426.

5. Karabegović, I.T.; Vukosavljević, P.V.; Novaković, M.M.; Gorjanović, S.Ž.; Džamić, A.M.; Lazić, M.L. Influence of the storage on bioactive compounds and sensory attributes of herbal liqueur. Dig. J. Nanomater. Biostruct. 2012, 7, 1587-1598.

6. Cornara, L.; La Rocca, A.; Marsili, S.; Mariotti, M.G. Traditional uses of plants in the eastern Riviera (Liguria, Italy). J. Ethnopharmacol. 2000, 125, 16-30. [CrossRef] [PubMed]

7. Gimeno Gasca, J.M. Tomillo (Thymus vulgaris L.). Med. Natur. 2001, 3, 173-175.

8. Alleman, F.; Gabriel, I.; Dufourcq, V.; Perrin, F.; Gabarrou, J.F. Utilisation des huiles essentielles en alimentation des volailles. 1. Perfornances de croussance et règlementation. INRA Prod. Anim. 2013, 26, 3-12.

9. Basch, E.; Ulbricht, C.; Hammerness, P.; Blevins, A.; Sollars, D. Thyme (Thymus vulgaris L.), thymol. J. Herb. Pharmacother. 2004, 4, 49-67. [CrossRef] [PubMed]

10. Duke, J.A. Handbook of Phytochemical Constituents of GRAS Herbs and Other Economic Plants; CRC Press: Boca Raton, FL, USA, 1992.

11. Tropicos.org. Missouri Botanical Garden. Available online: http://www.tropicos.org (accessed on 17 August 2016).

12. Thompson, J.D.; Chalchat, J.C.; Michet, A.; Linhart, Y.B.; Ehlers, B. Qualitative and quantitative variation on monoterpene co-occurrence and composition in the essential oil of Thymus vulgaris chemotypes. J. Chem. Ecol. 2003, 29, 859-880. [CrossRef] [PubMed]

13. Giordani, R.; Regli, P.; Kaloustian, J.; Mikaïl, C.; Abou, L.; Portugal, H. Antifungal effect of various essential oils against Candida albicans. Potentiation of antifungal action of amphotericin B by essential oil from Thymus vulgaris. Phytother. Res. 2004, 18, 990-995. [CrossRef] [PubMed]

14. Chizzola, R.; Michitsch, H.; Franz, C. Antioxidative properties of Thymus vulgaris leaves: Comparison of different extracts and essential oil chemotypes. J. Agric. Food Chem. 2008, 56, 6897-6904. [CrossRef] [PubMed]

15. Pavela, R.; Vrchotová, N.; Tř́ska, J. Mosquitocidal activities of thyme oils (Thymus vulgaris L.) against Culex quinquefasciatus (Diptera: Culicidae). Parasitol. Res. 2009, 105, 1365-1370. [CrossRef] [PubMed]

16. Adams, R.P. Identification of Essential Oil Components by Gas Chromatography/Mass Spectrometry, 4th ed.; Allured Publishing: Carol Stream, IL, USA, 2007.

17. Özek, T.; Tabanca, N.; Demirci, F.; Wedge, D.E.; Başer, K.H.C. Enantiomeric distribution of some linalool containing essential oils and their biological activities. Rec. Nat. Prod. 2010, 4, 180-192.

18. Shellie, R.; Marriott, P.; Cornwell, C. Application of comprehensive two-dimensional gas chromatography $(\mathrm{GC} \times \mathrm{GC})$ to the enantioselective analysis of essential oils. J. Sep. Sci. 2001, 24, 823-830. [CrossRef]

19. Bonaccorsi, I.; Sciarrone, D.; Cotroneo, A.; Mondello, L.; Dugo, P.; Dugo, G. Enantiomeric distribution of key volatile components in Citrus essential oils. Rev. Bras. Farmacog. 2011, 21, 841-849. [CrossRef]

20. Pragadheesh, V.S.; Saroj, A.; Yadav, A.; Samad, A. Chanitiya, C.S. Compositions, enantiomer characterization and antifungal activity of two Ocimum essential oils. Ind. Crops Prod. 2013, 50, 333-337. [CrossRef]

21. Tabanca, N.; Kirimer, N.; Demirci, B.; Demirci, F.; Başer, K.H.C. Composition and antimicrobial activity of the essential oils of Micromeria cristata subsp. phrygia and the enantiomeric distribution of borneol. J. Agric. Food. Chem. 2001, 49, 4300-4303. [CrossRef] [PubMed]

22. Imelouane, B.; Amhamdi, H.; Wathelet, J.P.; Ankit, M.; Khedid, K.; El Bachiri, A. Chemical composition and antimicrobial activity of essential oil of thyme (Thymus vulgaris) from eastern Morocco. Int. J. Agric. Biol. 2009, 11, 205-208.

23. Jordán, M.J.; Martínez, R.M.; Goodner, K.L.; Baldwin, E.A.; Sotomayor, J.A. Seasonal variation of Thymus hyemalis Lange and Spanish Thymus vulgaris L. essential oils composition. Ind. Crops Prod. 2006, 24, 253-263. [CrossRef] 
24. Bhaskara Reddy, M.V.; Angers, P.; Gosselin, A.; Arul, J. Characterization and use of essential oil from Thymus vulgaris against Botrytis cinerea and Rhizopus stolonifer in strawberry fruits. Phytochemistry 1998, 47, 1515-1520. [CrossRef]

25. Sacchetti, G.; Maietti, S.; Muzzoli, M.; Scaglianti, M.; Manfredini, S.; Radice, M.; Bruni, R. Comparative evaluation of 11 essential oils of different origin as functional antioxidants, antiradicals and antimicrobials in foods. Food Chem. 2005, 91, 621-632. [CrossRef]

26. Dimitrijević, S.I.; Mihajlovski, K.R.; Antonović, D.G.; Milanović-Stevanović, M.R.; Mijin, D.Ž. A study of the synergistic antilisterial effects of a sub-lethal dose of lactic acid and essential oils from Thymus vulgaris L., Rosmarinus officinalis L. and Origanum vulgare L. Food Chem. 2007, 104, 774-782. [CrossRef]

27. Gavahian, M.; Farahnaky, A.; Javidnia, K.; Majzoobi, M. Comparison of ohmic-assisted hydrodistillation with traditional hydrodistillation for the extraction of essential oils from Thymus vulgaris L. Innov. Food Sci. Emerg. Technol. 2012, 14, 85-91. [CrossRef]

28. Panizzi, L.; Flamini, G.; Cioni, P.L.; Morelli, I. Composition and antimicrobial properties of essential oils of four Mediterranean Lamiaceae. J. Ethnopharmacol. 1993, 39, 167-170. [CrossRef]

29. Lucchesi, M.E.; Chemat, F.; Smadja, J. Solvent-free microwave extraction of essential oil from aromatic herbs: Comparison with conventional hydro-distillation. J. Chromatogr. A 2004, 1043, 323-327. [CrossRef] [PubMed]

30. Zambonelli, A.; Zechini D’Aulerio, A.; Severi, A.; Benvenuti, S.; Maggi, L.; Bianchi, A. Chemical composition and fungicidal activity of commercial essential oils of Thymus vulgaris L. J. Essent. Oil Res. 2004, 16, 69-74. [CrossRef]

31. Hudaib, M.; Speroni, E.; Di Pietra, A.M.; Cavrini, V. GC/MS evaluation of thyme (Thymus vulgaris L.) oil composition and variations during the vegetative cycle. J. Pharmaceut. Biomed. Anal. 2002, 29, 691-700. [CrossRef]

32. Szczepanik, M.; Zawitowska, B.; Szumny, A. Insecticidal activities of Thymus vulgaris essential oil and its components (thymol and carvacrol) against larvae of lesser mealworm, Alphitobius diaperinus Panzer (Coleoptera: Tenebrionidae). Allelopath. J. 2012, 30, 129-142.

33. Bozin, B.; Mimica-Dukic, N.; Simin, N.; Anackov, G. Characterization of the volatile composition of essential oils of some Lamiaceae spices and the antimicrobial and antioxidant activities of the entire oils. J. Agric. Food Chem. 2006, 54, 1822-1828. [CrossRef] [PubMed]

34. Lee, S.J.; Umano, K.; Shibamoto, T.; Lee, K.G. Identification of volatile components in basil (Ocimum basilicum L.) and thyme leaves (Thymus vulgaris L.) and their antioxidant properties. Food Chem. 2005, 91, 131-137. [CrossRef]

35. Sartoratto, A.; Machado, A.L.M.; Delarmelina, C.; Figueira, G.M.; Duarte, C.T.; Rehder, V.L.G. Composition and antimicrobial activity of essential oils from aromatic plants used in Brazil. Braz. J. Microbiol. 2004, 35, 275-280. [CrossRef]

36. Dawidowicz, A.L.; Rado, E.; Wianowska, D.; Mardarowicz, M.; Gawdzik, J. Application of PLE for the determination of essential oil components from Thymus vulgaris L. Talanta 2008, 76, 878-884. [CrossRef] [PubMed]

37. Karami-Osboo, R.; Khodaverdi, M.; Ali-Akbari, F. Antibacterial effect of effective compounds of Satureja hortensis and Thymus vulgaris essential oils against Erwinia amylovora. J. Agric. Sci. Technol. 2010, 12, 35-45.

38. Al-Maqtary, M.A.A.; Alghalibi, S.M.; Alhamzy, E.H. Chemical composition and antimicrobial activity of essential oil of Thymus vulgaris from Yemen. Turk. J. Biochem. 2011, 36, 342-349.

39. Rota, M.C.; Herrera, A.; Martínez, R.M.; Sotomayor, J.A.; Jordán, M.J. Antimicrobial activity and chemical composition of Thymus vulgaris, Thymus zygis and Thymus hyemalis essential oils. Food Control 2008, 19, 681-687. [CrossRef]

40. Daferera, D.J.; Ziogas, B.N.; Polissiou, M.G. GC-MS analysis of essential oils from some Greek aromatic plants and their fungitoxicity on Penicillium digitatum. J. Agric. Food Chem. 2000, 48, 2576-2581. [CrossRef] [PubMed]

41. Nikolić, M.; Glamočlija, J.; Ferreira, I.C.F.R.; Calhelha, R.C.; Fernandes, Â.; Marković, T.; Marković, D.; Giweli, A.; Soković, M. Chemical composition, antimicrobial, antioxidant and antitumor activity of Thymus serpyllum L., Thymus algeriensis Boiss. and Reut and Thymus vulgaris L. essential oils. Ind. Crops Prod. 2014, 52, 183-190. [CrossRef] 
42. Soković, M.D.; Vukojević, J.; Marin, P.D.; Brkić, D.D.; Vajs, V.; van Griensven, L.J.L.D. Chemical composition of essential oils of Thymus and Mentha species and their antifungal activities. Molecules 2009, 14, 238-249. [CrossRef] [PubMed]

43. Ghasemi Pirbalouti, A.; Hashemi, M.; Taherian Ghahfarokhi, F. Essential oil and chemical compositions of wild and cultivated Thymus daenensis Celak and Thymus vulgaris L. Ind. Crops Prod. 2013, 48, 43-48. [CrossRef]

44. Baranauskienė, R.; Venskutonis, P.R.; Viškelis, P.; Dambrauskienè, E. Influence of nitrogen fertilizers on the yield and composition of thyme (Thymus vulgaris). J. Agric. Food Chem. 2003, 51, 7751-7758. [CrossRef] [PubMed]

45. Shabnum, S.; Wagay, M.G. Essential oil composition of Thymus vulgaris L. and their uses. J. Res. Dev. 2011, 11, 83-94.

46. Asllani, U.; Toska, V. Chemical composition of Albanian thyme oil (Thymus vulgaris L.). J. Essent. Oil Res. 2003, 15, 165-167. [CrossRef]

47. Porte, A.; Godoy, R.L.O. Chemical composition of Thymus vulgaris L. (thyme) essential oil from the Rio de Janeiro State (Brazil). J. Serbian Chem. Soc. 2008, 73, 307-310. [CrossRef]

48. Piccaglia, R.; Marotti, M. Composition of the essential oil of an Italian Thymus vulgaris L. ecotype. Flavour Fragr. J. 1991, 6, 241-244. [CrossRef]

49. Atti-Santos, A.C.; Pansera, M.R.; Paroul, N.; Atti-Serafini, L.; Moyna, P. Seasonal variation of essential oil yield and composition of Thymus vulgaris L. (Lamiaceae) from south Brazil. J. Essent. Oil Res. 2004, 16, 294-295. [CrossRef]

50. Golmakani, M.T.; Rezaei, K. Comparison of microwave-assisted hydrodistillation with the traditional hydrodistillation method in the extraction of essential oils from Thymus vulgaris L. Food Chem. 2008, 109, 925-930. [CrossRef] [PubMed]

51. Dawidowicz, A.L.; Rado, E.; Wianowska, D. Static and dynamic superheated water extraction of essential oil components from Thymus vulgaris L. J. Sep. Sci. 2009, 32, 3034-3042. [CrossRef] [PubMed]

52. Vurro, E.; Bruni, R.; Bianchi, A.; di Toppi, L.S. Elevated atmospheric $\mathrm{CO}_{2}$ decreases oxidative stress and increases essential oil yield in leaves of Thymus vulgaris grown in a mini-FACE system. Environ. Exp. Bot. 2009, 65, 99-106. [CrossRef]

53. McGimpsey, J.A.; Douglas, M.H.; van Klink, J.W.; Beauregard, D.A.; Perry, N.B. Seasonal variation in essential oil yield and composition from naturalized Thymus vulgaris L. in New Zealand. Flavour Fragr. J. 1994, 9 , 347-352. [CrossRef]

54. Mota, K.S.L.; Pereira, F.O.; de Oliveira, W.A.; Lima, I.O.; Lima, E.O. Antifungal activity of Thymus vulgaris L. essential oil and its constituent phytochemicals against Rhizopus oryzae: Interaction with ergosterol. Molecules 2012, 17, 14418-14433. [CrossRef] [PubMed]

55. Aziz, E.E.; Hendawi, S.T.; Azza, E.E.D.; Omer, E.A. Effect of soil type and irrigation intervals on plant growth, essential oil yield and constituents of Thymus vulgaris plant. Am.-Eurasian J. Agric. Environ. Sci. 2008, 4, 443-450.

56. Venskutonis, P.R. Effect of drying on the volatile constituents of thyme (Thymus vulgaris L.) and sage (Salvia officinalis L.). Food Chem. 1997, 59, 219-227. [CrossRef]

57. Šegvić Klarić, M.; Kosalec, I.; Masterlić, J.; Piecková, E.; Pepeljnak, S. Antifungal activity of thyme (Thymus vulgaris L.) essential oil and thymol against moulds from damp dwellings. Lett. Appl. Microbiol. 2007, 44, 36-42. [CrossRef] [PubMed]

58. Nguefack, J.; Tamgue, O.; Lekagne Dongmo, J.B.; Dakole, C.D.; Leth, V.; Vismer, H.F.; Amvam Zollo, P.H.; Nkengfack, A.E. Synergistic action between fractions of essential oils from Cymbopogon citratus, Ocimum gratissimum and Thymus vulgaris against Penicillium expansum. Food Control 2012, 23, 377-383. [CrossRef]

59. Grigore, A.; Paraschiv, I.; Colceru-Mihul, S.; Bubueanu, C.; Draghici, E.; Ichim, M. Chemical composition and antioxidant activity of Thymus vulgaris L. volatile oil obtained by two different methods. Rom. Biotechnol. Lett. 2010, 15, 5436-5443.

60. Díaz-Maroto, M.C.; Hidalgo, I.J.D.M.; Sánchez-Palomo, E.; Pérez-Coello, M.S. Volatile components and key odorants of fennel (Foeniculum vulgare Mill.) and thyme (Thymus vulgaris L.) oil extracts obtained by simultaneous distillation-extraction and supercritical fluid extraction. J. Agric. Food Chem. 2005, 53, 5385-5389. [CrossRef] [PubMed] 
61. Fachini-Queiroz, F.C.; Kummer, R.; Estevão-Silva, C.F.; Carvalho, M.D.B.; Cunha, J.M.; Grespan, R.; Bersani-Amado, C.A.; Cuman, R.K.N. Effects of thymol and carvacrol, constituents of Thymus vulgaris L. essential oil, on the inflammatory response. Evid.-Based Complement. Altern. Med. 2012, 2012, 657026. [CrossRef] [PubMed]

62. El-Nekeety, A.A.; Mohamed, S.R.; Hathout, A.S.; Hassan, N.S.; Aly, S.E.; Abdel-Wahhab, M.A. Antioxidant properties of Thymus vulgaris oil against aflatoxin-induced oxidative stress in male rats. Toxicon 2011, 57, 984-991. [CrossRef] [PubMed]

63. Pina-Vaz, C.; Gonçalves Rodrigues, A.; Pinto, E.; Costa-de-Oliveira, S.; Tavares, C.; Salgueiro, L.; Cavaleiro, C.; Gonçalves, M.J.; Martinez-de-Oliveira, J. Antifungal activity of Thymus oils and their major compounds. J. Eur. Acad. Dermatol. Venereol. 2004, 18, 73-78. [CrossRef] [PubMed]

64. Oh, K.; Matsuoka, H.; Sumita, O.; Takatori, K. Kurata, H. Automatic evaluation of antifungal volatile compounds on the basis of the dynamic growth process of a single hypha. Appl. Microbiol. Biotechnol. 1993, 38, 790-794. [CrossRef] [PubMed]

65. Anderson, A.R.; Wanner, K.W.; Trowell, S.C.; Warr, C.G.; Jaquin-Joly, E.; Zagatti, P.; Robertson, H.; Newcomb, R.D. Molecular basis of female odorant responses in Bombyx mori. Insect Biochem. Mol. Biol. 2009, 39, 189-197. [CrossRef] [PubMed]

66. Liu, F.; Chen, L.; Appel, A.G.; Liu, N. Olfactory responses of the antennal trichoid sensilla to chemical repellents in the mosquito, Culex quinquefasciatus. J. Insect Physiol. 2013, 59, 1169-1177. [CrossRef] [PubMed]

67. Prasse, A.; Siehl, H.U.; Zeller, K.P.; Berger, S.; Sicker, D. Wie unsere Nase die enantiomeren Linaloole unterscheidet. Chem. Unserer Zeit 2014, 48, 342-353. [CrossRef]

68. Asztemborska, M.; Ochocka, J.R. Chiral monoterpenoids in plants-Enantioselective chromatographic analysis, and their bioactivity. Stud. Nat. Prod. Chem. 2002, 27, 361-391. [CrossRef]

69. De Sousa, D.P.; Nóbrega, F.F.; Santos, C.C.; de Almeida, R.N. Anticonvulsant activity of the linalool enantiomers and racemate: Investigation of chiral influence. Nat. Prod. Commun. 2010, 5, 1847-1851. [PubMed]

70. Da Silva, A.C.R.; Lopes, P.M.; de Azevedo, M.M.B.; Costa, D.C.M.; Alviano, C.S.; Alviano, D.S. Biological activities of $\alpha$-pinene and $\beta$-pinene enantiomers. Molecules 2012, 17, 6305-6316. [CrossRef] [PubMed]

71. Lis-Balchin, M.; Ochocka, R.J.; Deans, S.G.; Asztemborska, M.; Hart, S. Differences in bioactivity between the enantiomers of $\alpha$-pinene. J. Essent. Oil Res. 1999, 11, 393-397. [CrossRef] 